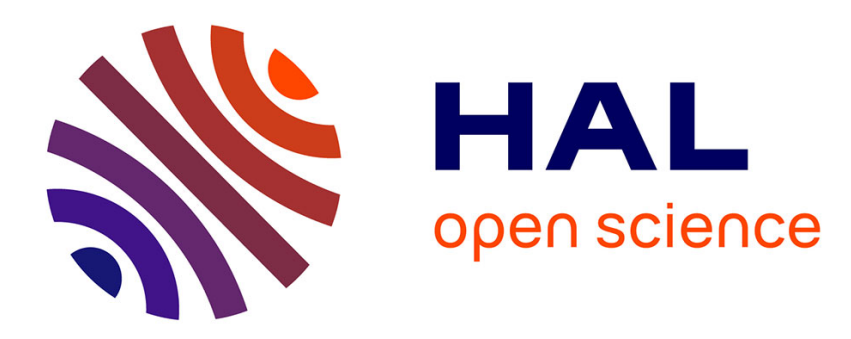

\title{
Information Systems: Towards a System of Information Systems
}

Majd Saleh, Marie-Helene Abel

\section{To cite this version:}

Majd Saleh, Marie-Helene Abel. Information Systems: Towards a System of Information Systems. KMIS 2015 7th International Conference on Knowledge Management and Information Sharing, Nov 2015, Lisbonne, Portugal. pp.193-200, 10.5220/0005596101930200 . hal-01282774

\section{HAL Id: hal-01282774 https://hal.science/hal-01282774}

Submitted on 7 Mar 2016

HAL is a multi-disciplinary open access archive for the deposit and dissemination of scientific research documents, whether they are published or not. The documents may come from teaching and research institutions in France or abroad, or from public or private research centers.
L'archive ouverte pluridisciplinaire HAL, est destinée au dépôt et à la diffusion de documents scientifiques de niveau recherche, publiés ou non, émanant des établissements d'enseignement et de recherche français ou étrangers, des laboratoires publics ou privés. 


\title{
Information Systems: Towards a System of Information Systems
}

\author{
Majd Saleh and Marie-Hélène Abel \\ Sorbonne Universités, Université de Technologie de Compiègne, CNRS, UMR 7253 Heudiasyc, Compiègne, France \\ \{majd.saleh,marie-helene.abel $\} @ u t c . f r$
}

Keywords: Information Systems, Knowledge Management, System of Systems, System of Information Systems

\begin{abstract}
Information Systems are viewed as a set of services creating a workflow of information directed to specific groups and members. This allows individuals to share ideas and their talents with other members. In such manner, tasks can be carried out both efficiently and effectively. Due to the nature of Information Systems that revolves around creating information useful to users, and in some higher forms of Information Systems creating knowledge, management of information and/or knowledge is part of their functionalities. In this paper we aim to study the placement of Information Systems as part of a System of Systems (SoS), as these large systems poses significant technical improvement in terms of information interoperability that overcomes conceptual and technical barriers. Therefore, we move towards defining and modeling System of Information Systems (SoIS). This paper discovers what is currently known about Information Systems and Systems of Systems, and proceeds towards suggesting an architecture of a System of Information Systems that integrates several Information Systems and allows information to be transferred at ease between those different components.
\end{abstract}

\section{INTRODUCTION}

Information Systems have begun to turn simplicity to complexity, and to turn hard-to-use to easy-to-use. However, effectiveness and efficiency on information systems is not satisfying (An, 2010). This universal nature of existing Information Systems has generated a strong interest in using an existing set of systems as the basis for a System of Systems (Simpson and Dagli, 2008). Further interest in the System of Systems approach is stimulated by rapid development, deployment and expansion of new and existing systems. While successful system and System of Systems production provides the basis of great potentials, many system development activities result in failure. The issue is that while many individual systems work well as an independent system, they fail when incorporated as a component of a System of Systems. The required System of Systems will need to connect systems that cross organizational boundaries, come from multiple domains, and generates an overwhelming amount of information. Users struggle to deal with the information produced by each Information System independently by traversing through these systems and keeping track of the generated information separately. A solution might be found in a System of Systems that operates as a single entry point for sev- eral Information Systems granting the user access to information produced from multiple Information Systems, and providing the ability to aggregate available services to even create an added value not possible to maintain when those systems were operating separately. In other words, we might look at this new system as a System of Information Systems.

Our objective from this paper is to propose an architecture of a System of Information Systems comprised of independent Information Systems, with minimum changes required for the new SoIS to operate successfully, and provide unified entry point to information and services with an extra added value not present in the separate Information Systems.

This paper is organized as follows: section 2 will provide a use case scenario of the intended SoIS to highlight the need of such system. Then, the notion of System of Systems is defined and a discussion of the differences between System of Systems and Composite Systems is presented to draw clear line between these two concepts. After that, in section 4, the SoIS architectural model is presented and then discussed by defining the concept of SoIS, describing the different information systems involved in our SoIS, and presenting an example following our architectural model of SoIS. Later, we discuss our findings in section 5 by addressing the issue of expanding our SoIS archi- 
tectural model to accept further information systems, and compare it to existing SoS. Finally, we conclude with section 6 .

\section{USE CASE SCENARIO}

- There is a growing need not only to use Information Systems, but also to integrate them with other systems that can benefit from the information generated and shared within them. Furthermore, services provided by other systems can have positive impact on the use of Information Systems.

To put the idea in perspective, let us take the following scenario; Say a journalist is investigating a certain topic. First the journalist might visit various Social Media Networks and search for this topic and the people involved in it. Next, the journalist might need to unveil the ambiguity on certain aspects to better understand the topic, so he/she starts using Wikipedia (Wikipedia, 2015) or DBpedia (DBpedia, 2015) to search for keywords related to this topic, or even use the archive system from other databases to search for information. Later on, the journalist might publish some of his/her findings on various Social Media Networks to see the public reaction to the topic under investigation. Of course, not to forget that the journalist needs to store and keep track of the information he/she collected from different Information Systems, and be able to access the information at all times to reform an encompassing idea about the topic at hand. To sum things up, we mentioned a scenario where the user surfed several Information Systems and collected information from those systems separately. The user also had to work with the information collected from separate systems collectively. The struggle is with accessing all these different Information Systems and keeping track of the information obtained and created while doing the work required. The problems we face here reside in the time required to traverse between different systems and keep track, organize, and archive all the information obtained from these systems.

\section{SYSTEM OF SYSTEMS}

In this section we plan to go through various definitions of System of Systems presented in the literature. Then, we are going to highlight the differences between Systems of Systems and Composite Systems in order to be clear when describing our own System of Systems.

\subsection{Definitions}

The notion of System of Systems (SoS) can be viewed as an evolution of the standard notion of systems. Here, we aim to provide abstract and generic definitions of terminology involved in the domain of systems.

As mentioned by (Karcanias and Hessami, 2010) "A system is an interconnection organization of objects which are embedded in a given environment". An object is defined as "a general unit (abstract or physical) defined in terms of its attributes and the possible relations between them". Furthermore, these objects are embedded in an environment. For a given object, we define its environment as the "set of objects, signals, events, structures, which are considered topologically external to the object, and are linked to the object in terms of a structure, relations between their attributes". Briefly, a system can be defined as a relationship mapped over a set of objects in an encompassing environment (Simpson and Dagli, 2008).

Many definitions of a SoS exist. An aggregate of systems leads to the creation of new forms of systems which may be either described within the framework of composite systems, or demonstrate additional features which add complexity to the description and may be referred to as System of Systems. We can summarize the definitions mentioned in literature as the following:

Definition (i): Systems of Systems exist when there is a presence of a majority of the following five characteristics: operational and managerial independence, geographic distribution, emergent behavior, and evolutionary development (Jamshidi, 2011).

Definition (ii): Systems of Systems are largescale concurrent and distributed systems that are comprised of complex systems (Jamshidi, 2011) (Carlock and Fenton, 2001).

Definition (iii): Enterprise Systems of Systems Engineering is focused on coupling traditional systems engineering activities with enterprise activities of strategic planning and investment analysis (Carlock and Fenton, 2001).

Definition (iv): System of Systems Integration is a method to pursue development, integration, interoperability, and optimization of systems to enhance performance in future battlefield scenarios (Carlock and Fenton, 2001).

Definition (v): System of systems is a collection of task-oriented or dedicated systems that pool their resources and capabilities together to obtain a new, more complex, 'meta-system' which offers more functionality and performance than simply the sum of the constituent systems (Manthorpe, 1996). 
Definition (vi): System of systems is a set of different elements connected or related so as to perform a unique function not performable by the elements alone (Rechtin and Maier, 2000).

In the light of the mentioned definitions we can describe the notion of System of Systems (SoS) as the following; Systems of systems are large-scale integrated systems which are heterogeneous and independently operable on their own, but are networked together for a common goal. The goal, as mentioned before, may be cost, performance, robustness, etc. In other terms, A System of Systems is a super system comprised of other elements which themselves are independent complex operational systems and interact among themselves to achieve a common goal. Each element of a SoS achieves well-substantiated goals even if they are detached from the rest of the SoS.

\subsection{System of Systems Versus Composite Systems}

The definitions listed in the previous section are mostly descriptive, but they capture crucial features of what a generic definition should involve; however, they do not answer the question, why is this new notion different than that of composite systems? The distinctive feature of the notion of System of Systems ( $\mathrm{SoS})$ is that it is considered as an evolution of the standard notion in engineering of Composite Systems (CoS) (Sage and Cuppan, 2001) (Karcanias, 2008).

Developing the transition from $\mathrm{CoS}$ to $\mathrm{SoS}$ we need to identify the commonalities and differences between the two notions. We note:

- Both CoS and SoS are compositions of simpler objects, or systems.

- Both CoS and SoS are embedded in the environment of a larger system.

- The objects, or sub-systems in CoS do not have their independent goal, they are not autonomous and their behavior is subject to the rules of the interconnection topology.

- The interconnection rule in $\mathrm{CoS}$ is expressed as a graph topology.

- The subsystems in SoS may have their own goals and some of them may be autonomous, semiautonomous, or organized as autonomous groupings of composite systems.

- There may be a connection rule expressed as a graph topology for the information structures of the subsystems in a SoS.
- The SoS is linked to an environment where every subsystem enters as an agent with their individual Operational Set, Goals.

The evolution of SoS and its ability to solve problems which cannot be addressed by the traditional view of $\mathrm{CoS}$ comes from these three major factors:

- Autonomy: the systems involved in SoS are at least partially autonomous

- Local views: no system has a full global view of the SoS.

- Decentralization: there is no designated single controlling system, but decision and information gathering is distributed.

\section{SYSTEM OF INFORMATION SYSTEMS}

In this section we propose a new system that combines the services from different Information Systems. First, we are going to define our new system as a System of Information Systems (SoIS). Then, we are going to present a generic architecture of the SoIS. Finally we propose an initial design for the SoIS that can serve as an example of the architecture.

\subsection{Definition}

The notion of System of Information Systems is defined by (Carlsson and Stankiewicz, 1991) as "networks of agents interacting in a specific technology area under a particular institutional infrastructure for the purpose of creating, diffusing, and utilizing technology focused on knowledge, information, and competence flow." (Breschi and Malerba, 1996) describe SoIS as "the specific clusters of the firms, technologies, and industries involved in the generation and diffusion of new technologies and in the knowledge flow that takes place among them."

Based on the definitions provided we can summarize the features of SoIS as follows:

- SoIS addresses the impact of the interrelationships between different SoS.

- SoIS is concerned with the flow of information and knowledge among different information systems.

- SoIS is responsible for generating information from the emergent SoS.

- Information interoperability is a key issue when designing a SoIS. 


\subsection{Architecture}

In this part we are going to present the generic architecture of the SoIS. As seen in (Fig. 1), the SoIS will be able to connect to several Information Systems (System A, System B etc.). These systems are working separately. Each of which has its own services and databases. Introducing new services to these Information systems can be carried out by the SoIS. These new services follow the MEMORAe approach. As defined by (Ala Atrash, 2014), MEMORAe approach is to manage heterogeneous information resources within organizations. The approach is comprised of a semantic model (called MEMORAe-core 2) and a web platform (called MEMORAe) which is based on the semantic model. The model and the platform make together a support to enhance the process of resources management. The purpose behind introducing MEMORAe approach to the SoIS is to provide the added value for other systems comprising the SoIS by the resources management means of MEMORAe.

In general, the SoIS is represented as a group of services and a database. The database will hold information about the users' account for the different Information Systems comprising the SoIS. On the other hand, the services residing in the SoIS can either be directed to add systems to the orchestration, or emerging services created from the aggregation of different Information Systems with the MEMORAe approach. In addition, the two headed arrows linking the SoIS with the Information systems represent information path between the SoIS and the different Information Systems.

\subsection{Example}

As mentioned earlier, the SoIS is composed of several Information Systems. In this part we will take four Information Systems to create a SoIS from them by following the architecture presented earlier in (Fig. 1). These systems are Twitter, Semantic Hashtag, DBpedia, and MEMORAe. First we will take a look at each of these systems separately and highlight their functionality as independent systems, before unveiling their role as parts of the SoIS.

Twitter: Its characteristics are the short messages that people can post to the system. With a maximum of 140 characters users may transcribe immediate information. These messages are called "tweets" and the novel expression "to tweet" stands for posting a message on Twitter. Originally used for exchanging ordinary information, Twitter is also used during political campaigns or political debates, for business communication (Zhao and Rosson, 2009).
In Twitter people update their activities with friends simply sending text messages, sometimes enriched with photos, links and hash tags. The \# symbol, is used to mark keywords or topics in a Tweet. It was created organically by Twitter users as a way to categorize messages (Potts et al., 2011). The hashtag is used to categorize tweets and help users to search more easily in Twitter search. Hash-tagged words that become very popular are often trending topics.

Semantic Hashtag (Lai et al., 2013): The web interface of this system provides an input box for writing the text of the tweet. The user may input a free text as in usual Twitter clients. The constraint of 140 characters is handled by a counter. In addition to the free text, a driven process allows to insert one or more semantic hashtags. For this purpose the user inserts a meaningful keyword concerning the text of the tweet. Such a keyword is used to discover within a knowledge base the identifiers of resources associated to the keyword itself. In this prototype we use the knowledge base available in the DBpedia project (Auer et al., 2007), the semantic web mirror of Wikipedia (Wikipedia, 2015). DBpedia provides a web service called Lookup Service3. The service discovers and returns DBpedia URIs from related keywords and concerning the resources contained in the DBpedia knowledge base.

MEMORAe: As defined by (Ala Atrash, 2014), MEMORAe approach is to manage heterogeneous information resources within organizations. The approach is comprised of a semantic model (called MEMORAe-core 2) and a web platform (called MEMORAe) which is based on the semantic model. The model and the platform make together a support to enhance the process of organizational learning.

The MEMORAe project uses the Semantic Web standards, therefore, the ontologies occurring in the system are written in OWL. Users registered in the MEMORAe system can access one or more knowledge bases. When a base is chosen, a user can view a semantic map of concepts related to the selected base. Then, a user can create and share resources around the concepts of the map.

After being introduced to the components of our SoIS, let us take a look at the first draft of these Information Systems working together as SoIS.

First we are going list down the important services provided by each system. This list is available in (Table. 1).

After listing down all key services from the Information Systems under study, we can write down the list of service we expect to have by aggregating those systems in SoIS. The list of services available by the SoIS is present in (Table. 2). 


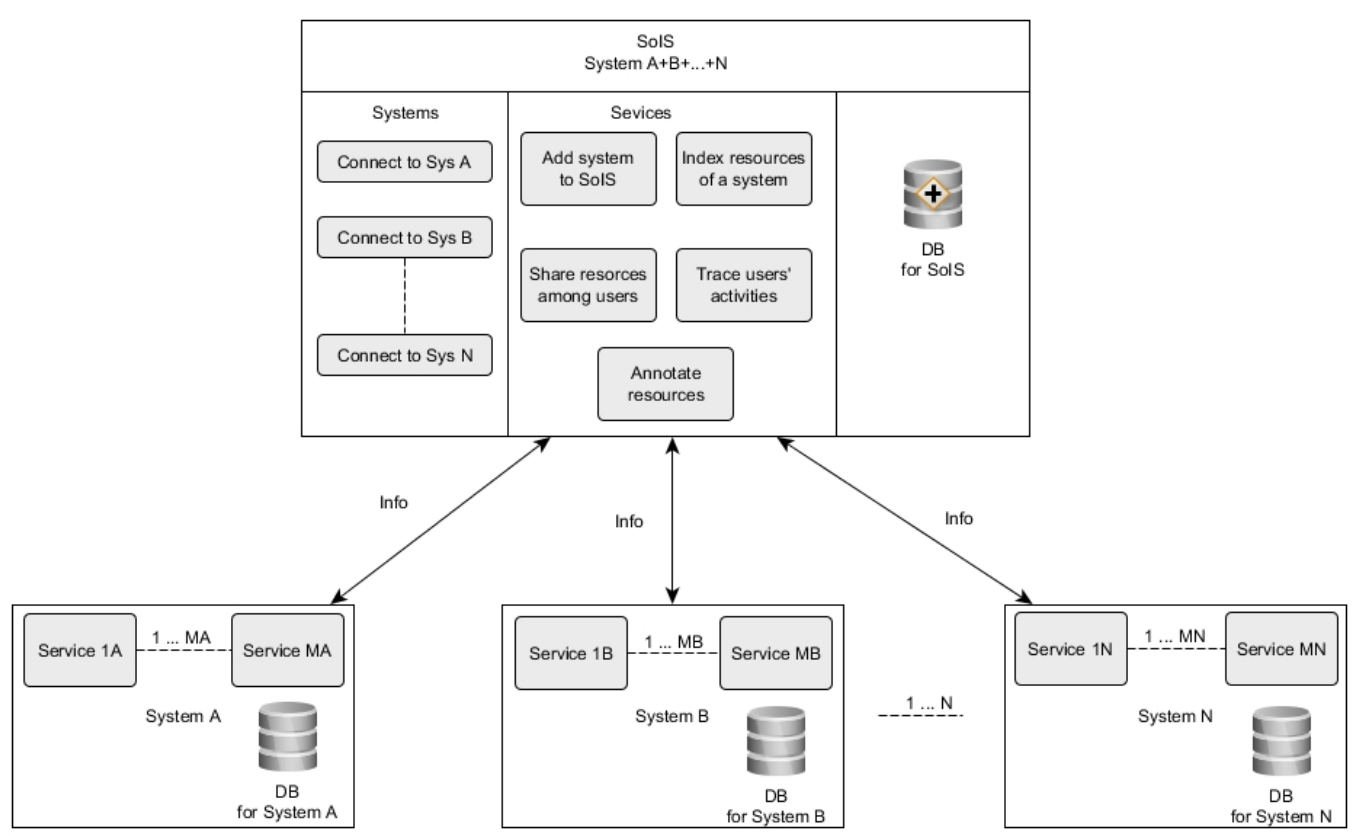

Figure 1: Architectural model of the SoIS.

\begin{tabular}{|l|l|l|l|l|}
\cline { 2 - 5 } \multicolumn{1}{c|}{} & \multicolumn{1}{c|}{$\begin{array}{c}\text { Twitter } \\
\text { (System A) }\end{array}$} & \multicolumn{1}{c|}{$\begin{array}{c}\text { Semantic Hashtag } \\
\text { (System B) }\end{array}$} & \multicolumn{1}{c|}{$\begin{array}{c}\text { MEMORAe } \\
\text { (System C) }\end{array}$} & \multicolumn{1}{c|}{$\begin{array}{c}\text { DBPedia } \\
\text { (System D) }\end{array}$} \\
\hline \multirow{4}{*}{$\begin{array}{l}\text { Available } \\
\text { Services }\end{array}$} & $\begin{array}{l}\text { Share information as } \\
\text { tweet }\end{array}$ & Post on Twitter & Capitalize knowledge & Capitalize knowledge \\
\cline { 2 - 5 } & Add hashtag to tweets & $\begin{array}{l}\text { Use Semantic Hashtags from } \\
\text { DBPedia in tweets }\end{array}$ & $\begin{array}{l}\text { Index resources by a } \\
\text { semantic map }\end{array}$ & Lookup Service \\
\cline { 2 - 5 } & & View Twitter feed & & \\
\cline { 2 - 5 } & $\begin{array}{l}\text { View the description of the } \\
\text { semantic hashtag }\end{array}$ & & \\
\hline
\end{tabular}

Table 1: Available services in the Information Systems comprising the SoIS.

To better understand the functionalities of this SoIS, we can start by explaining how the application Semantic Hashtag works. This application allows users to create semantic tweets. In more details, the user may input a free text as in usual Twitter clients. The constraint of 140 characters is handled by the application. In addition to the free text, a driven process allows to insert one or more semantic hashtags. For this purpose the user inserts a meaningful keyword concerning the text of the tweet. This keyword is used to discover the identifiers of resources associated to the keyword itself. The application uses the ontology provided by DBpedia. The semantic hashtag is created from the ontology URI related to the selected resource and automatically inserted in the text of the tweet (X). An added value can be achieved by allowing the semantic hashtag to use ontologies other than the one provided by Dbpedia. In other words, to expand the scope of the semantic hashtag application to knowledge base systems other than DBpedia.
In addition, we might also think of allowing the information shared by Twitter as tweets to be indexed semantically in a knowledge base system.

The idea behind our work is as follows; we intend to design a system that combines services from other systems providing an added value for users. This added value could not be obtained when the intended systems were performing separately. There is Twitter that allows us to share resources as tweets. Also, there is the Semantic Hashtag system that allows users to create tweets with semantic hashtags created using a knowledge base available by DBpedia. We also have MEMORAe, a web application that goes beyond simple content management system, and allows users to index resources semantically over a map of concepts. The scenario we propose is of a system that allows users to create tweets with semantic hashtag from the knowledge base available in both DBpedia and MEMORAe and be able to index the tweets semantically in MEMORAe. 
The mockup design of the SoIS is shown in (Fig. 2). In this illustration we can easily see the simplicity in design in order to make user experience friendly and pleasant. We can note the following features available in this design:

- The user will be provided with login credentials to access the SoIS interface.

- On the left panel, the user can manage the different accounts for the different Information Systems available in the SoIS. At this point the user can access both his/her Twitter and MEMORAe accounts.

- On the middle top panel we can see the module from the Semantic Hashtag application where the user is able to create text tweets with semantic hashtags. These semantic hashtags can be either generated from the knowledge base available in DBpedia or MEMORAe. Here we note an added value as the users of the Semantic Hashtag application were able to generate the hashtags semantically from DBpedia only.

- On the middle bottom panel we can view Twitter feed from the users Twitter account.

- On the right panel the user can use MEMORAe to index and store tweets semantically. The user can select a concept from his/her MEMORAe account and a sharing space to make this tweet available by it. Then the user can index the selected tweet according to the selected concept and sharing space. This is also an added value to the SoIS, as users were not able to index tweets in MEMORAe before.

Next we are going to present a simple scenario to create a semantic hashtag using the knowledge base from MEMORAe in the SoIS. We will use standard UML sequence diagram to represent the sequence of actions taking place to perform this service in the SoIS. Sequence diagrams are good means to represent interactions in such complex systems as mentioned by (Majd Saleh, 2015). The sequence diagram is shown in (Fig. 3).

\section{DISCUSSION}

In the previous section we presented the SoIS with an example that will grant users access to various Information Systems from a single interface. The questions here are: Can we add more Information Systems to this SoIS? Is it limited to these Information Systems only?

To answer those questions we can look at the functionality that allows us to generate semantic hashtags

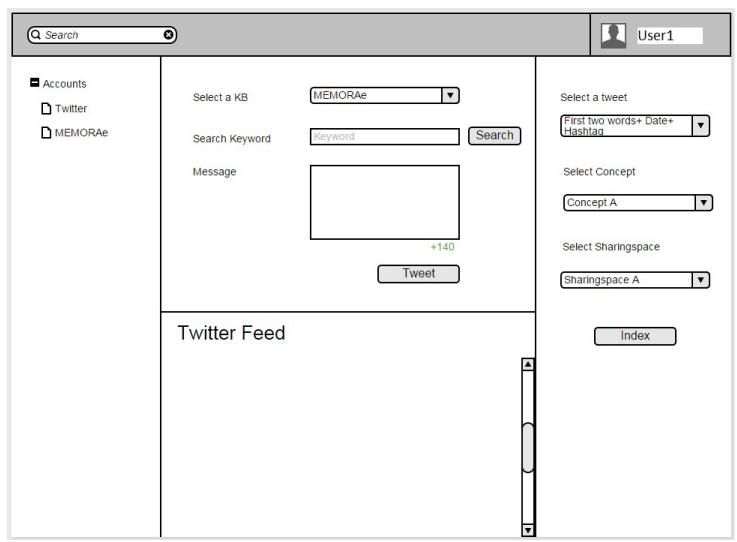

Figure 2: Example of the SoIS.

\begin{tabular}{|l|l|}
\cline { 2 - 2 } \multicolumn{1}{c|}{} & \multicolumn{1}{c|}{$\begin{array}{c}\text { System of Information Systems } \\
\text { (System A+B+C+D) }\end{array}$} \\
\hline \multirow{4}{*}{$\begin{array}{l}\text { Available } \\
\text { Services }\end{array}$} & $\begin{array}{l}\text { Add accounts } \\
\text { (Twitter and MEMORAe for starter) }\end{array}$ \\
\cline { 2 - 2 } & $\begin{array}{l}\text { Use Semantic Hashtags } \\
\text { from DBPedia orMEMORAe in tweets }\end{array}$ \\
\cline { 2 - 2 } & View Twitter feed \\
\cline { 2 - 2 } & $\begin{array}{l}\text { View the description of the semantic } \\
\text { hashtag other resources indexed by }\end{array}$ \\
\hline
\end{tabular}

Table 2: Available services in the SoIS.

and post them on Twitter. At first, the user was able to create semantic hashtag from the knowledge base provided by DBpedia. This functionality was made available by DBpedia system through the lookup service that allows searching its knowledge base using keywords, therefore, returning results corresponding to those keywords. In this SoIS we were able expand this functionality and allow users to search MEMORAes knowledge base through a similar lookup service made available by MEMORAe. In such manner, we can include other Information Systems to the searchable knowledge base if they provide such service. Furthermore, the SoIS provide the functionality of indexing tweets in MEMORAe. The numerous types of resources handled by MEMORAe, varying from documents, notes, and forum entry to even weblinks, allows it index almost any resource we can think of. For example, we might consider using MEMORAe in this SoIS to index users emails from an email system or calendar entry from his/her agenda application. In this manner, the SoIS can grow to handle new Information Systems as the need to use such systems grows. However, we discussed the expansion of the architecture presented earlier to accept new Information Systems, but what about including other SoS and SoIS to be part of a greater SoIS. This raises the concern of whether to include a SoIS as a whole 


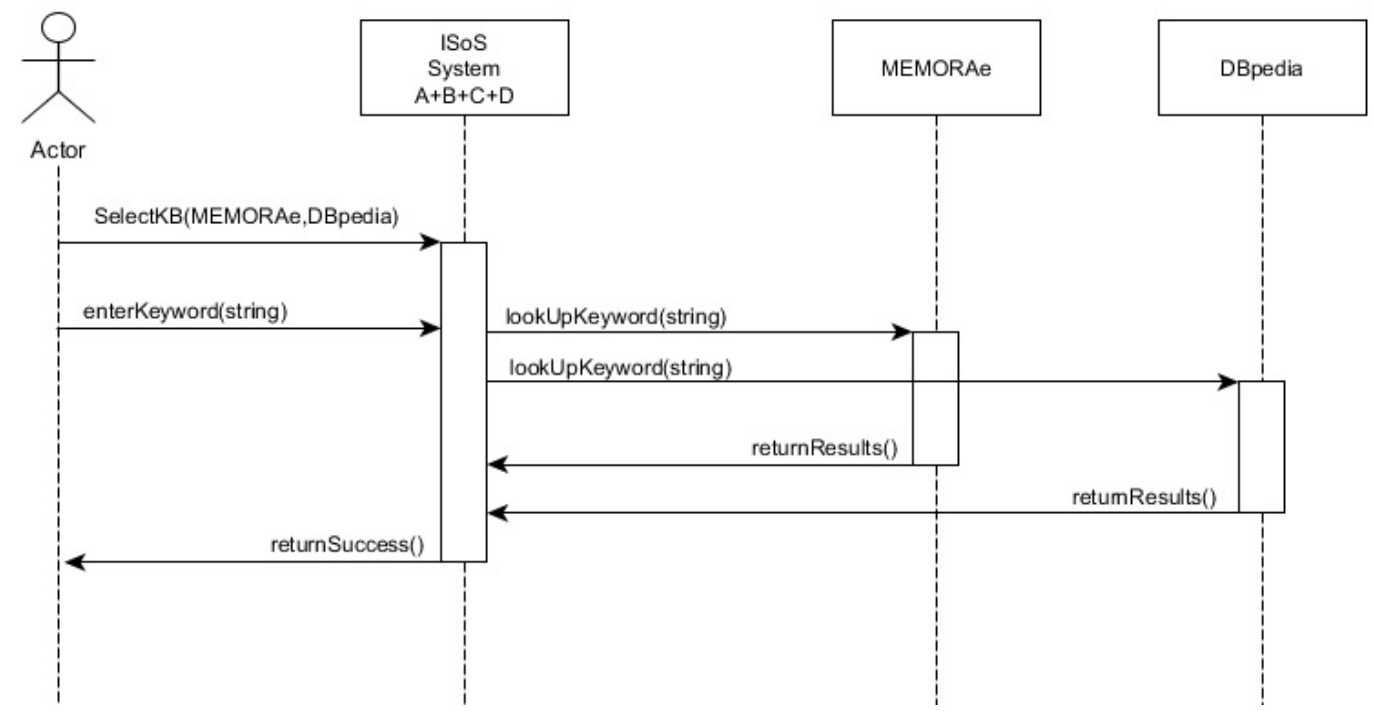

Figure 3: Sequence diagram for creating semantic hashtage using the knowledge base from MEMORAe and DBpedia.

in a more encompassing SoIS, or only include parts of it as separate Information Systems. We can argue that our architecture permit the SoIS to include services from many Information Systems. In such manner, we could consider the composite SoIS as a whole to be part of an encompassing SoIS, or only include parts of it as separate Information Systems.

It is also worth mentioning that the new SoIS presented in this paper can be compared to other SoS in the market. For example, we can take the Paraimpu SoS (Paraimpu, 2015). Paraimpu is a social tool with the aim to allow people to connect, compose and share things, services and devices to create personalized applications on Internet (of Things). It allows the user to create Sensors, Actuators and Connections between them in the Paraimpu workspace. The Connection can be configured in relation to different scenarios. These Sensors and Actuators represent different systems coming together in Paraimpu SoS to provide new services to users based on the connection created between them. A scenario might be where the user add a "Mount Washington Wind Speed Sensor", which, every 30 minutes, measures the wind speed in that world location, where the biggest wind speed was ever measured. Then, the user adds a "Twitter Actuator". Finally the user connects the Sensor to the Twitter Actuator. The connection will be configured in order to post a tweet under certain conditions based on the Sensors readings. The design of this SoS is simple, where you have separate panels for Sensors, Actuators, and connections. The user can drag and drop the components to create connections between various systems and make benefit from their interactions. In a similar way, our SoIS is design with sim- plicity in mind to give users the ability to interact with different Information Systems and benefit from their interactions for an added value not present when those systems were operating separately.

\section{CONCLUSION}

Our goal was to take current Information Systems and move towards System of Information Systems (SoIS) to aggregate services and exchange information with simplicity and ease. To achieve this goal we undertake this research to determine what is currently known about Information Systems, System of Systems, and System of Information Systems in the literature, and deploy this knowledge to propose an example of a SoIS composed of various Information Systems. First, we proposed a use case scenario to illustrate the need of such SoIS. Then we defined the notion of SoS and highlighted the differences between $\mathrm{SoS}$ and $\mathrm{CoS}$ to make it clear that the new system can be considered a SoS not a CoS. After that, we moved forward to define the notion of SoIS and describe the various Information Systems comprising this SoIS. Finally, we proposed an example of a SoIS.

We found potential to the SoIS to expand and hold new Information Systems. It was also clear that combining services from various Information Systems will result in an added value to users not present when those systems were operating separately. The example of the SoIS is also found to be similar in comparison to other SoS in this field.

The next step is to expand our work and introduce new Information Systems to the SoIS based on the 
example presented in this study and users needs. It should also be tested in a real world experiment. The SoIS should keep simple interface, with all the services as far from the user as a single click, to keep the users experience useful and friendly.

\section{Acknowledgement}

This project is done under ECOPACK project and funded by ANR-ASTRID programme.

\section{REFERENCES}

Ala Atrash, Marie-Hélène Abel, C. M. (2014). Supporting organizational learning with collaborative annotation. In 6th international conference on knowledge management and information sharing (KMIS), pages 237- 244 .

An, H. (2010). Research on information systems problems based on an ecological angle. In Challenges in Environmental Science and Computer Engineering (CESCE), 2010 International Conference on, volume 1, pages 281-284. IEEE.

Auer, S., Bizer, C., Kobilarov, G., Lehmann, J., Cyganiak, R., and Ives, Z. (2007). Dbpedia: A nucleus for a web of open data. Springer.

Breschi, S. and Malerba, F. (1996). Sectoral innovation systems: technological regimes, Schumpeterian $d y$ namics and spatial boundaries. Centro studi sui processi di internazionalizzazione, Università commerciale'Luigi Bocconi'.

Carlock, P. G. and Fenton, R. E. (2001). System of systems (sos) enterprise systems engineering for information-intensive organizations. Systems engineering, 4(4):242-261.

Carlsson, B. and Stankiewicz, R. (1991). On the nature, function and composition of technological systems. Journal of evolutionary economics, 1(2):93-118.

DBpedia (2015). Dbpdia. [Online; accessed 20-May-2015].

Jamshidi, M. (2011). System of systems engineering: innovations for the twenty-first century, volume 58. John Wiley \& Sons.

Karcanias, N. (2008). Structure evolving systems and control in integrated design. Annual reviews in Control, 32(2):161-182.

Karcanias, N. and Hessami, A. (2010). Complexity and the notion of system of systems: Part (ii): defining the notion of system of systems. In World Automation Congress (WAC), 2010, pages 1-7. IEEE.

Lai, C., Moulin, C., and Abel, M.-H. (2013). Management of twitter resources in a semantic organizational memory. In P2P, Parallel, Grid, Cloud and Internet Computing (3PGCIC), 2013 Eighth International Conference on, pages 695-700. IEEE.
Majd Saleh, Marie-Hélène Abel, V. M. (2015). Investigating the similarity between collaboration systems and digital ecosystems. In 19th IEEE International Conference on Computer Supported Cooperative Work in Design.

Manthorpe, W. H. (1996). The emerging joint system of systems: A systems engineering challenge and opportunity for apl. Johns Hopkins APL Technical Digest, 17(3):305.

Paraimpu (2015). paraimpu. [Online; accessed 20-May2015].

Potts, L., Seitzinger, J., Jones, D., and Harrison, A. (2011). Tweeting disaster: hashtag constructions and collisions. In Proceedings of the 29th ACM international conference on Design of communication, pages 235240. ACM.

Rechtin, E. and Maier, M. W. (2000). The art of systems architecting. CRC Press.

Sage, A. P. and Cuppan, C. D. (2001). On the systems engineering and management of systems of systems and federations of systems. Information, Knowledge, Systems Management, 2(4):325-345.

Simpson, J. J. and Dagli, C. H. (2008). System of systems: Power and paradox. In System of Systems Engineering, 2008. SoSE'08. IEEE International Conference on, pages 1-5. IEEE.

Wikipedia (2015). Wikipedia, the free encyclopedia. [Online; accessed 20-May-2015].

Zhao, D. and Rosson, M. B. (2009). How and why people twitter: the role that micro-blogging plays in informal communication at work. In Proceedings of the ACM 2009 international conference on Supporting group work, pages 243-252. ACM. 